\title{
Constructing the covariant three-point vertices systematically
}

\author{
Seong Youl Choi $\odot^{*}$ and Jae Hoon Jeong ${ }^{\dagger}$ \\ Department of Physics and RIPC, Jeonbuk National University, Jeonju 54896, Korea
}

(Received 28 November 2021; accepted 2 January 2022; published 19 January 2022)

\begin{abstract}
An algorithm is developed for efficiently constructing the Lorentz covariant effective three-point vertices of the decay of a particle into two daughter particles in which all the masses and spins of the three particles can be arbitrary. The closely related one-to-one correspondence between the helicity formalism and the covariant formulation is exploited for counting the number of independent terms and identifying the basic covariant three-point vertices. Assembling the basic operators according to the developed algorithm enables us to construct all the covariant three-point vertices systematically.
\end{abstract}

DOI: 10.1103/PhysRevD.105.016016

\section{INTRODUCTION}

Despite the completion of the Standard Model (SM) [1] of particle physics through the Higgs-boson discovery at the Large Hadron Collider (LHC) [2], various unsolved conceptual issues and unexplained experimental observations suggest the SM to be an effective theory of a more fundamental theory. One powerful strategy for probing new physics beyond the SM (BSM) is to keep our studies as model-independent as possible and to search for new particles by including spins higher than unity.

Various high-spin (composite) hadrons have been discovered and investigated [3]. A gravitino appears as a spin-3/2 supersymmetric partner of the massless spin-2 graviton in supergravity [4]. The gravitational-wave discovery [5] indicates the existence of massless spin-2 gravitons at the quantum level. The massive spin-2 particles as the Kaluza-Klein (KK) excitations of the massless graviton have been studied in extra-dimension models [6]. Recently, the scenario of high-spin dark matter (DM) particles has been investigated intensively [7]. For studying all these aspects, it is crucial to probe all the allowed effective interactions of particles of any spin as well as lowspin SM particles in a model-independent way.

In this work, we develop an efficient algorithm for systematically constructing all the effective three-point vertices consistent with Lorentz invariance and locality. If necessary, other symmetry principles like local gauge invariance or Bose/Fermi symmetries may be invoked.

\footnotetext{
sychoi@jbnu.ac.kr

jaehoonjeong229@gmail.com
}

Published by the American Physical Society under the terms of the Creative Commons Attribution 4.0 International license. Further distribution of this work must maintain attribution to the author(s) and the published article's title, journal citation, and DOI. Funded by SCOAP ${ }^{3}$.
Specifically, we consider the decay of a massive particle of spin $J$ and mass $m$ into a massive particle $M_{1}$ of spin $s_{1}$ and mass $m_{1}$ and a massive antiparticle $\bar{M}_{2}$ of spin $s_{2}$ and mass $m_{2}$, while commenting how the massless case with $m_{1}=0$ or $m_{2}=0$ can be accommodated straightforwardly. This study is a natural generalization of two previous works having dealt with the identical-spin particles of zero equal mass [8] and nonzero equal mass [9].

In this development, we adopt the conventional description of integer and half-integer wave tensors [10] and utilize the closely related equivalence between the helicity formalism in the Jacob-Wick (JW) convention [11] and the standard covariant formulation. Their one-to-one correspondence enables us to identify every basic building block for constructing the covariant three-point vertex corresponding to every helicity combination explicitly. We note in passing that another powerful procedure for constructing the general three-point vertices is to use a spinor formalism [12].

\section{CHARACTERIZATION IN THE HELICITY FORMALISM}

The helicity formalism [11] allows us to efficiently describe the two-body decay of a particle $X$ of spin $J$ and mass $m$ into a particle $M_{1}$ of spin $s_{1}$ and mass $m_{1}$ and an antiparticle $\bar{M}_{2}$ of spin $s_{2}$ and mass $m_{2}$. For the sake of a transparent analysis, we describe the two-body decay $X \rightarrow M_{1} \bar{M}_{2}$ in the $X$ rest frame $(X \mathrm{RF})$

$$
X(p, \sigma) \rightarrow M_{1}\left(k_{1}, \lambda_{1}\right)+\bar{M}_{2}\left(k_{2}, \lambda_{2}\right),
$$

with their momenta, $\left\{p, k_{1}, k_{2}\right\}$, and helicities, $\left\{\sigma, \lambda_{1}, \lambda_{2}\right\}$, as depicted in the left side of Fig. 1.

In the JW convention [11], the helicity amplitude of the decay $X \rightarrow M_{1} \bar{M}_{2}$ is decomposed as 

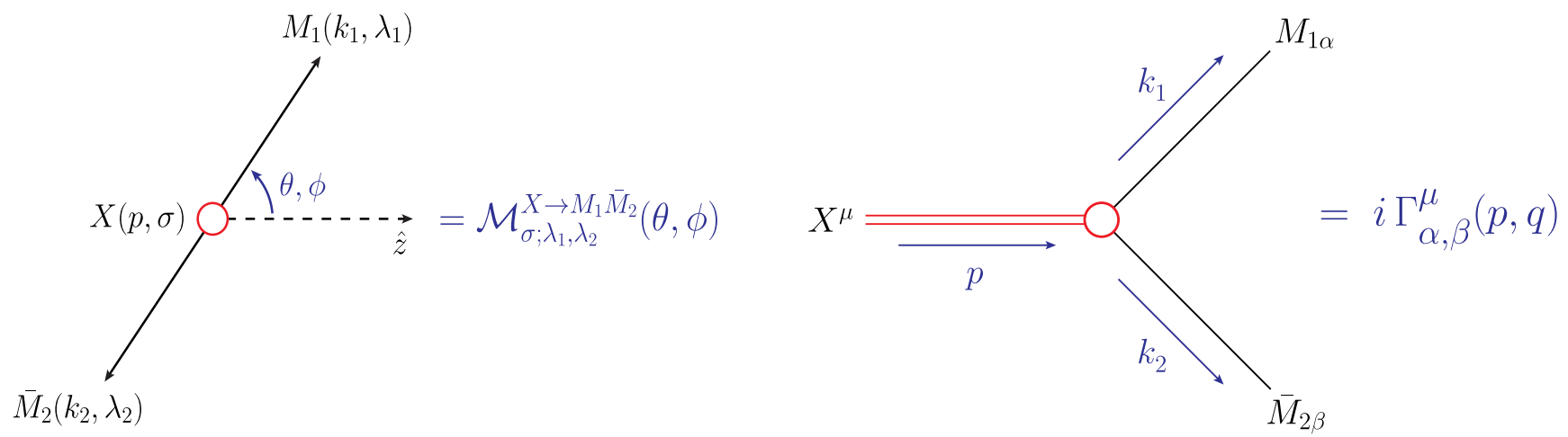

FIG. 1. Left: kinematic configuration for the helicity amplitude $\mathcal{M}_{\sigma ; \lambda_{1}, \lambda_{2}}^{X \rightarrow M_{1} \bar{M}_{2}}(\theta, \phi)$ of the two-body decay $X \rightarrow M_{1} \bar{M}_{2}$ of a massive particle $X$ into a particle $M_{1}$ and an antiparticle $\bar{M}_{2}$ in the $X \mathrm{RF}$. The polar and azimuthal angles, $\theta$ and $\phi$, are defined with respect to an appropriately chosen coordinate system in the $X \mathrm{RF}$. Right: general $X M_{1} \bar{M}_{2}$ covariant three-point vertex $\Gamma_{\alpha, \beta}^{\mu}(p, q)$ for the decay of a particle $X$ of spin $J$ and mass $m$ into a particle $M_{1}$ of spin $s_{1}$ and mass $m_{1}$ and an antiparticle $\bar{M}_{2}$ of spin $s_{2}$ and mass $m_{2}$. The indices, $\mu, \alpha$ and $\beta$, stand for the sequences of $\mu=\mu_{1} \cdots \mu_{n}, \alpha_{1} \cdots \alpha_{n_{1}}$ and $\beta_{1} \cdots \beta_{n_{2}}$ collectively with the non-negative integer $n=J$ or $n=J-1 / 2$ in the integer or half-integer spin $J$ case and the non-negative integers $n_{1,2}=s_{1,2}$ or $n_{1,2}=s_{1,2}-1 / 2$ in the integer or half-integer spin $s_{1,2}$ case, respectively. The symmetric and antisymmetric momentum combinations, $p=k_{1}+k_{2}$ and $q=k_{1}-k_{2}$, are introduced for the sake of notation.

$$
\begin{aligned}
\mathcal{M}_{\sigma ; \lambda_{1}, \lambda_{2}}^{X \rightarrow M_{1} \bar{M}_{2}}(\theta, \phi)= & \mathcal{C}_{\lambda_{1}, \lambda_{2}}^{J} d_{\sigma, \lambda_{1}-\lambda_{2}}^{J}(\theta) e^{i\left(\sigma-\lambda_{1}+\lambda_{2}\right) \phi} \\
& \text { with } \quad\left|\lambda_{1}-\lambda_{2}\right| \leq J,
\end{aligned}
$$

in terms of the polar and azimuthal angles, $\theta$ and $\phi$, defining the $M_{1}$ momentum direction in a fixed $X \mathrm{RF}$ coordinate system (see the left side of Fig. 1), where the reduced helicity amplitudes $\mathcal{C}_{\lambda_{1}, \lambda_{2}}^{J}$ do not depend on the $X$ helicity $\sigma$ due to rotational invariance and the polar-angle dependence is fully encoded in the Wigner $d$ function $d_{\sigma, \lambda_{1}-\lambda_{1}}^{J}(\theta)$ given in the convention of Rose [13]. The helicity $\sigma$ of the spin- $J$ massive particle $X$ takes one of $2 J+1$ values between $-J$ and $J$. On the other hand, the helicities $\lambda_{1,2}$ of the spin- $s_{1,2}$ massive particles, $M_{1}$ and $\bar{M}_{2}$, can take one of $2 s_{1,2}+1$ values between $-s_{1,2}$ and $s_{1,2}$, under the constraint $\left|\lambda_{1}-\lambda_{2}\right| \leq J$. The number $n\left[J, s_{1}, s_{2}\right]$ of independent reduced helicity amplitudes are given by

$$
n\left[J, s_{1}, s_{2}\right]= \begin{cases}\left(2 s_{1}+1\right)\left(2 s_{2}+1\right) & \text { for } J \geq s_{1}+s_{2}, \\ \left(2 s_{1}+1\right)\left(2 s_{2}+1\right)-\left(s_{1}+s_{2}-J\right)\left(s_{1}+s_{2}-J+1\right) & \text { for }\left|s_{1}-s_{2}\right| \leq J<s_{1}+s_{2}, \\ \left(s_{1}+s_{2}-\left|s_{1}-s_{2}\right|+1\right)(2 J+1) & \text { for } J<\left|s_{1}-s_{2}\right|,\end{cases}
$$

that is valid for any allowed combinations of the spins $J$ and $s_{1,2}$ if the particles, $M_{1}$ and $\bar{M}_{2}$, are massive.

The master key to the algorithm for constructing the general three-point vertices is to find every helicity-specific operator generating a reduced helicity amplitude nonzero only for each specific helicity combination of $\left[\lambda_{1}, \lambda_{2}\right]$. In the present work, we find all the helicity-specific operators for the two-body decay $X \rightarrow M_{1} \bar{M}_{2}$ in the three cases, (iii), $(i h h)$, and (hhi), with the indices, $i$ and $h$, indicating whether the spins of $X, M_{1}$ and $\bar{M}_{2}$ are integer or halfinteger, respectively. The helicity-specific operators for the other processes such as those in the case (hih) and for the charge-conjugated processes can be obtained by converting the derived ones according to simple symmetry arguments, as described explicitly in Sec. V B.

\section{BOSONIC AND FERMIONIC WAVE TENSORS}

Generically, the decay amplitude of a particle $X$ of spin $J$ and mass $m$ into a particle $M_{1}$ of spin $s_{1}$ and mass $m_{1}$ and an antiparticle $\bar{M}_{2}$ of spin $s_{2}$ and mass $m_{2}$ can be written in terms of the covariant three-point vertex tensor $\Gamma$ (see the right side of Fig. 1 for its diagrammatic description) as

$$
\mathcal{M}_{\sigma ; \lambda_{1}, \lambda_{2}}^{X \rightarrow M_{1} \bar{M}_{2}}=\bar{\psi}_{1}^{\alpha_{1} \cdots \alpha_{n_{1}}}\left(k_{1}, \lambda_{1}\right) \Gamma_{\alpha_{1} \cdots \alpha_{n_{1}}, \beta_{1} \cdots \beta_{n_{2}}}^{\mu_{1} \cdots \mu_{n}}(p, q) \psi_{2}^{\beta_{1} \cdots \beta_{n_{2}}}\left(k_{2}, \lambda_{2}\right) \psi_{\mu_{1} \cdots \mu_{n}}(p, \sigma),
$$


with the non-negative integer $n=J$ or $n=J-1 / 2$ in the integer or half-integer spin $J$ case and the non-negative integers $n_{1,2}=s_{1,2}$ or $n_{1,2}=s_{1,2}-1 / 2$ in the integer or half-integer spin $s_{1,2}$ case, respectively. Here, $\left\{p, k_{1}, k_{2}\right\}$ and $\left\{\sigma, k_{1}, k_{2}\right\}$ are the momenta and helicities of the particles $X, M_{1}$, and $\bar{M}_{2}$. The momenta, $p=k_{1}+k_{2}$ and $q=k_{1}-k_{2}$, are symmetric and antisymmetric under the interchange of $k_{1} \leftrightarrow k_{2}$.

We show the explicit expressions of the wave tensors of an on-shell particle $X$ of spin $J$, mass $m$, momentum $p$, and helicity $\sigma$ [10]. The wave tensors of the particles $M_{1}$ and $\bar{M}_{2}$ can be obtained by substituting $s_{1,2}, m_{1,2}, k_{1,2}$, and $\lambda_{1,2}$ for $J, m, p$, and $\sigma$ from the expressions given in the following.

The bosonic particle $X$ of an integer spin $J=n$ with a non-negative integer $n$ is represented by a rank- $n$ wave tensor $\epsilon_{\mu_{1} \cdots \mu_{n}}(p, \sigma)$ as

$$
\begin{aligned}
\psi_{\mu_{1} \cdots \mu_{n}}(p, \sigma)= & \epsilon_{\mu_{1} \cdots \mu_{n}}(p, \sigma) \\
= & \sqrt{\frac{2^{n}(n+\sigma) !(n-\sigma) !}{(2 n) !}} \\
& \times \sum_{\{\tau\}=-1}^{1} \delta_{\tau_{1}+\cdots+\tau_{n}, \sigma} \prod_{i=1}^{n} \frac{\epsilon_{\mu_{i}}\left(p, \tau_{i}\right)}{\sqrt{2}^{\left|\tau_{i}\right|}},
\end{aligned}
$$

with the convention $\{\tau\}=\tau_{1}, \ldots, \tau_{n}$, which satisfies the on-shell condition $\left(p^{2}-m^{2}\right) \epsilon_{\mu_{1} \cdots \mu_{n}}(p, \sigma)=0$ for any helicity value of $\sigma$ taking an integer value between $-n$ and $n$. The bosonic wave tensor (3.2) satisfies

$$
\begin{aligned}
\varepsilon_{\alpha \beta \mu_{i} \mu_{j}} & \epsilon^{\mu_{1} \cdots \mu_{i} \cdots \mu_{j} \cdots \mu_{n}}(p, \sigma)=0, \\
g_{\mu_{i} \mu_{j} \mu_{j}} \epsilon^{\mu_{1} \cdots \mu_{i} \cdots \mu_{j} \cdots \mu_{n}}(p, \sigma) & =0, \\
p_{\mu_{i}} \epsilon^{\mu_{1} \cdots \mu_{i} \cdots \mu_{n}}(p, \sigma) & =0,
\end{aligned}
$$

as it is totally symmetric, traceless, and divergence-free in the four-vector indices. If the mass $m=0$, the wave tensor has only two maximal-magnitude helicities $\sigma= \pm n= \pm J$ and its form is given simply by a direct product of $n$ spin- 1 wave vectors, each of which carries the same helicity of \pm 1 .

On the other hand, the fermionic particle $X$ of a halfinteger spin $J=n+1 / 2$ with a noninteger value $n$ is represented in terms of the spin- $1 / 2 u$ and $v$ Dirac spinors of a particle and an antiparticle. The fermionic particle and antiparticle wave spinors are given by [10]

$$
\begin{aligned}
\psi_{\mu}(p, \sigma) & =u_{\mu_{1} \cdots \mu_{n}}(p, \sigma) \\
& =\sum_{\tau= \pm 1 / 2} \sqrt{\frac{J+2 \tau \sigma}{2 J}} \epsilon_{\mu_{1} \cdots \mu_{n}}(p, \sigma-\tau) u(p, \tau),
\end{aligned}
$$

$$
\begin{aligned}
\psi_{\mu}(p, \sigma) & =v_{\mu_{1} \cdots \mu_{n}}(p, \sigma) \\
& =\sum_{\tau= \pm 1 / 2} \sqrt{\frac{J+2 \tau \sigma}{2 J}} \epsilon_{\mu_{1} \cdots \mu_{n}}^{*}(p, \sigma-\tau) v(p, \sigma),
\end{aligned}
$$

where the spin- $1 / 2$ spinors satisfy their own on-shell conditions $(\not p-m) u\left(p, \pm \frac{1}{2}\right)=0$ and $(\not p+m) v\left(p, \pm \frac{1}{2}\right)=0$. We note that the massive wave spinors are totally symmetric, traceless, and divergence-free in the four-vector indices, as well. In the helicity amplitude (3.1), the fermionic $M_{1}$ wave spinor is given by $\bar{\psi}_{1}^{\alpha}\left(k_{1}, \lambda_{1}\right)=\psi_{1}^{\alpha \dagger}\left(k_{1}, \lambda_{1}\right) \gamma^{0}$. If the mass $m=0$, the $X$ wave spinor has two maximal-magnitude helicities $\pm J$ and its form is given simply by a product of a $u$ or $v$ spinor and $n$ spin- 1 wave vectors with $n=J-1 / 2$.

For a kinematic description of the decay $X \rightarrow M_{1} \bar{M}_{2}$ in the $X \mathrm{RF}$, we introduce three unit vectors expressed in terms of the polar and azimuthal angles, $\theta$ and $\phi$ as

$$
\begin{aligned}
& \hat{n}=(\sin \theta \cos \phi, \sin \theta \sin \phi, \cos \theta), \\
& \hat{\theta}=(\cos \theta \cos \phi, \cos \theta \sin \phi,-\sin \theta), \\
& \hat{\phi}=(-\sin \phi, \cos \phi, 0),
\end{aligned}
$$

being mutually orthonormal, i.e., $\hat{n} \cdot \hat{\theta}=\hat{\theta} \cdot \hat{\phi}=\hat{\phi} \cdot \hat{n}=0$ and $\hat{n} \cdot \hat{n}=\hat{\theta} \cdot \hat{\theta}=\hat{\phi} \cdot \hat{\phi}=1$. In addition, we express the four-momentum sum $p=k_{1}+k_{2}$ and the four-momentum difference $q=k_{1}-k_{2}$ as

$$
p=m \hat{p} \quad \text { and } \quad q=m\left(\omega_{1}^{2}-\omega_{2}^{2}\right) \hat{p}+m \kappa \hat{k},
$$

in terms of two dimensionless rescaled masses $\omega_{1,2}=$ $m_{1,2} / m$ and the kinematic factor $\kappa=\eta^{+} \eta^{-}$with $\eta^{ \pm}=\sqrt{1-\left(\omega_{1} \pm \omega_{2}\right)^{2}}$. Here, the two orthonormal dimensionless four vectors $\hat{p}$ and $\hat{k}$ are given by

$$
\hat{p}=(1, \overrightarrow{0}) \quad \text { and } \quad \hat{k}=(0, \hat{n})
$$

in the $X \mathrm{RF}$. These normalized momenta $\hat{p}$ and $\hat{k}$, along with a few rescaled mass-dependent kinematic factors, can be exploited for expressing all the reduced helicity amplitudes in the XRF.

We adopt the JW convention [11] for deriving the spin-1 vectors and spin- $1 / 2$ spinors in the $X$ RF. The spin- 1 wave vectors for the particle $X$ with momentum $p$ and two particles, $M_{1}$ and $\bar{M}_{2}$, whose momenta $k_{1,2}=(p \pm q) / 2=$ $m\left(e_{1,2}, \pm \kappa \hat{n}\right) / 2$ with $e_{1,2}=1 \pm\left(\omega_{1}^{2}-\omega_{2}^{2}\right)$, are given in the $X \mathrm{RF}$ by

$\epsilon(p, \pm 1)=\frac{1}{\sqrt{2}}(0, \mp 1,-i, 0), \quad \epsilon(p, 0)=(0,0,0,1)$, 


$$
\begin{aligned}
\epsilon_{1}\left(k_{1}, \pm 1\right) & =\frac{1}{\sqrt{2}} e^{ \pm i \phi}(0, \mp \hat{\theta}-i \hat{\phi}), \\
\epsilon_{1}\left(k_{1}, 0\right) & =\frac{1}{2 \omega_{1}}\left(\kappa, e_{1} \hat{n}\right), \\
\epsilon_{2}\left(k_{2}, \pm 1\right) & =\frac{1}{\sqrt{2}} e^{\mp i \phi}(0, \pm \hat{\theta}-i \hat{\phi}), \\
\epsilon_{2}\left(k_{2}, 0\right) & =\frac{1}{2 \omega_{2}}\left(-\kappa, e_{2} \hat{n}\right),
\end{aligned}
$$

satisfying the relation $\epsilon_{2}\left(k_{2}, \pm 1\right)=\epsilon_{1}\left(k_{1}, \mp 1\right)=$ $-\epsilon_{1}^{*}\left(k_{1}, \pm 1\right)=-\epsilon_{2}^{*}\left(k_{2}, \mp 1\right)$. On the other hand, the spin-1/2 $u$ spinor of the particle fermion $X$ is given in the $X \mathrm{RF}$ by

$$
\begin{aligned}
u\left(p, \pm \frac{1}{2}\right) & =\sqrt{m}\left[\begin{array}{l}
\xi_{ \pm}(\hat{z}) \\
\xi_{ \pm}(\hat{z})
\end{array}\right] \text { with } \\
\xi_{+}(\hat{z}) & =\left[\begin{array}{l}
1 \\
0
\end{array}\right] \quad \text { and } \quad \xi_{-}(\hat{z})=\left[\begin{array}{l}
0 \\
1
\end{array}\right],
\end{aligned}
$$

and the spin-1/2 $u_{1}$ and $v_{2}$ spinors of the particle and antiparticle fermions $M_{1}$ and $\bar{M}_{2}$ are given by

$$
\begin{aligned}
& u_{1}\left(k_{1}, \pm \frac{1}{2}\right)=\sqrt{\frac{m}{2}}\left[\begin{array}{c}
\sqrt{e_{1} \mp \kappa} \chi_{ \pm}(\hat{n}) \\
\sqrt{e_{1} \pm \kappa} \chi_{ \pm}(\hat{n})
\end{array}\right], \\
& v_{2}\left(k_{2}, \pm \frac{1}{2}\right)=\sqrt{\frac{m}{2}}\left[\begin{array}{c} 
\pm \sqrt{e_{2} \pm \kappa} \chi_{ \pm}(\hat{n}) \\
\mp \sqrt{e_{2} \mp \kappa} \chi_{ \pm}(\hat{n})
\end{array}\right],
\end{aligned}
$$

where the 2-component spinors $\chi_{ \pm}(\hat{n})$ are written in terms of the angles, $\theta$ and $\phi$, as

$\chi_{+}(\hat{n})=\left[\begin{array}{l}\cos \frac{\theta}{2} \\ \sin \frac{\theta}{2} e^{i \phi}\end{array}\right]$ and $\chi_{-}(\hat{n})=\left[\begin{array}{c}-\sin \frac{\theta}{2} e^{-i \phi} \\ \cos \frac{\theta}{2}\end{array}\right]$,

being orthonormal, i.e., $\chi_{a}^{\dagger}(\hat{n}) \chi_{b}(\hat{n})=\delta_{a, b}$, with $a, b= \pm$ in the $X \mathrm{RF}$.

\section{BASIC COVARIANT THREE-POINT VERTICES}

In this section, we find all the Lorentz-covariant basic bosonic and fermionic three-point operators by deriving the helicity-specific operators corresponding to the reduced helicity amplitudes for the three spin combinations of $\left(J, s_{1}, s_{2}\right)=(1,1,1), \quad(1,1 / 2,1 / 2) \quad$ and $(1 / 2,1 / 2,1)$ explicitly. The set of all these operators is the backbone for constructing the covariant three-point vertices.

\section{A. Bosonic vertex operators}

First, we consider the $1 \rightarrow 1+1$ two-body decay of a spin-1 particle $X$ into two spin-1 massive vector bosons, $M_{1}$ and $\bar{M}_{2}$. The number of independent terms including the
$1 \rightarrow 1+1$ decay is $n[1,1,1]=7$, accounting for the seven reduced helicity amplitudes, $\mathcal{C}_{0,0}^{1}, \mathcal{C}_{0, \pm 1}^{1}, \mathcal{C}_{ \pm 1,0}^{1}$, and $\mathcal{C}_{ \pm 1, \pm 1}^{1}$, in the $X \mathrm{RF}$. After a little manipulation, we find the five covariant three-point vertex operators

$$
\begin{aligned}
U_{\alpha \beta}^{0} \hat{k}_{\mu} & =\hat{p}_{1 \alpha} \hat{p}_{2 \beta} \hat{k}_{\mu} \leftrightarrow \mathcal{C}_{0,0}^{1}=\kappa^{2}, \\
U_{1 \alpha \mu}^{ \pm} \hat{p}_{2 \beta} & =\frac{1}{2}\left[g_{\perp \alpha \mu} \pm i\langle\alpha \mu \hat{p} \hat{k}\rangle\right] \hat{p}_{2 \beta} \leftrightarrow \mathcal{C}_{ \pm 1,0}^{1}=\kappa, \\
U_{2 \beta \mu}^{ \pm} \hat{p}_{1 \alpha} & =\frac{1}{2}\left[g_{\perp \beta \mu} \mp i\langle\beta \mu \hat{p} \hat{k}\rangle\right] \hat{p}_{1 \alpha} \leftrightarrow \mathcal{C}_{0, \pm 1}^{1}=-\kappa,
\end{aligned}
$$

and the two covariant composite operators $U^{ \pm}$of the contraction of the basic operators $U_{1}^{ \pm}$and $U_{2}^{ \pm}$satisfying

$$
\begin{aligned}
U_{\alpha \beta}^{ \pm} \hat{k}_{\mu} & \equiv g^{\mu_{1} \mu_{2}} U_{1 \alpha \mu_{1}} U_{2 \beta \mu_{2}} \hat{k}_{\mu} \\
& =\frac{1}{2}\left[g_{\perp \alpha \beta} \pm i\langle\alpha \beta \hat{p} \hat{k}\rangle\right] \hat{k}_{\mu} \leftrightarrow \mathcal{C}_{ \pm 1, \pm 1}^{1}=-1,
\end{aligned}
$$

expressed with two rescaled momenta $\hat{p}_{1,2}=2 \omega_{1,2} \hat{p}$ vanishing for $m_{1,2}=0$, the orthogonal tensor $g_{\perp \mu \nu}=$ $g_{\mu \nu}-\hat{p}_{\mu} \hat{p}_{\nu}+\hat{k}_{\mu} \hat{k}_{\nu}$ and $\langle\mu \nu \hat{p} \hat{k}\rangle=\varepsilon_{\mu \nu \rho \sigma} \hat{p}^{\rho} \hat{k}^{\sigma}$ defined in terms of the totally antisymmetric Levi-Civita tensor with the convention $\varepsilon_{0123}=+1$. Each of the seven covariant three-point vertices generates solely its corresponding reduced helicity amplitude, as shown in Eqs. (4.1), (4.2), (4.3), and (4.4).

In order to clarify the essential role of each of the bosonic basic operators, let us introduce an integer-helicity lattice space consisting of $\left(2 s_{1}+1\right) \times\left(2 s_{2}+1\right)$ in order for each point $\left[\lambda_{1}, \lambda_{2}\right]$ to stand for its corresponding reduced helicity amplitude $\mathcal{C}_{\lambda_{1}, \lambda_{2}}^{J}$ existing only when $\left|\lambda_{1}-\lambda_{2}\right| \leq J$ and $\left|\lambda_{1,2}\right| \leq s_{1,2}$. We can deduce that the one-step horizontal and vertical transitions are dictated by the basic operators, $U_{1}^{ \pm}$and $U_{2}^{ \pm}$, from the point $\left[\lambda_{1}, \lambda_{2}\right]$ to the point $\left[\lambda_{1} \pm 1, \lambda_{2}\right]$ and the point $\left[\lambda_{1}, \lambda_{2} \pm 1\right]$ in the helicity-lattice space, respectively, as shown in the left panel of Fig. 2. The transitions by the basic operators, $U_{1}^{ \pm}$and $U_{2}^{ \pm}$, enable us to deduce that the one-step diagonal transitions are dictated by the composite operators $U^{ \pm}$from the point $\left[\lambda_{1}, \lambda_{2}\right]$ to the point $\left[\lambda_{1} \pm 1, \lambda_{2} \pm 1\right]$. On the other hand, the three operators, $\hat{k}$, and $\hat{p}_{1,2}$, forming the operator $U_{0}$ and multiplied by the basic and composite operators generate no transition, that is to say, the helicity point remains intact by the three momentum operators, solely changing the number of $\mu, \alpha$ and $\beta$ four-vector indices. Properly combining the six bosonic raising and lowering operators, $U_{1}^{ \pm}, U_{2}^{ \pm}$, and $U^{ \pm}$, along with the three rescaled momentum operators, $\hat{k}$ and $\hat{p}_{1,2}$, enables us to reach every integer-helicity lattice point. To summarize, for any given integer $J$ and integer $s_{1,2}$, we can weave the covariant three-point vertex corresponding to every integer-helicity combination of $\left[\lambda_{1}, \lambda_{2}\right]$ efficiently and systematically. The explicit form of every 

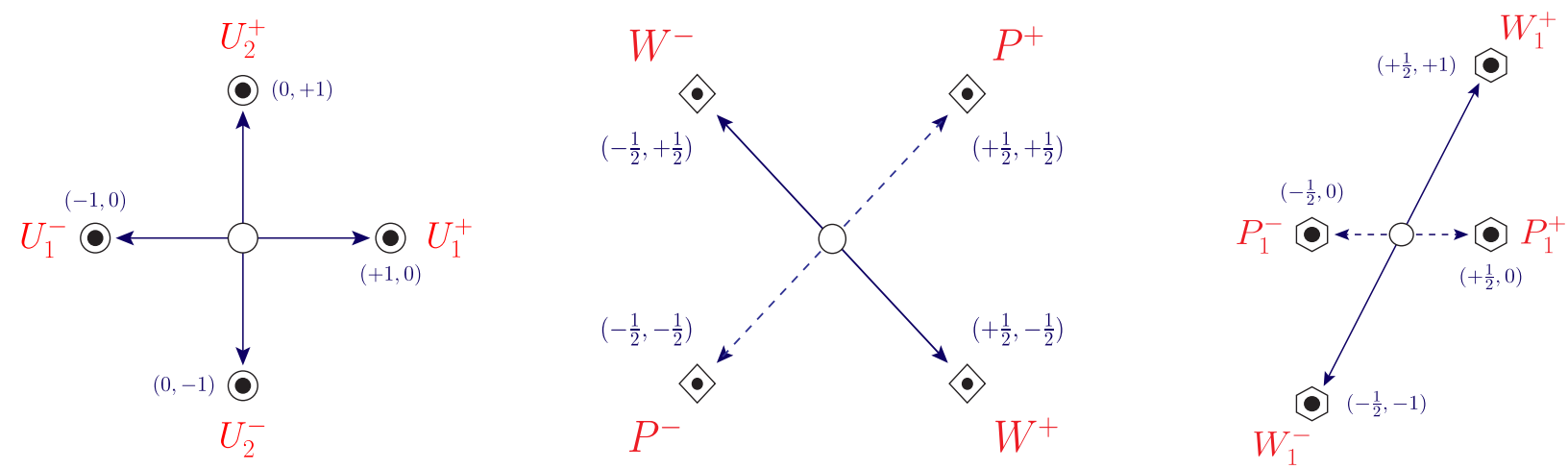

FIG. 2. Diagrammatic description of the four basic bosonic $U_{1}^{ \pm}$and $U_{2}^{ \pm}$operators on the left-hand side, the four basic fermionic $P^{ \pm}$and $W^{ \pm}$operators in the middle, and the four basic fermionic operators $P_{1}^{ \pm}$and $W_{1}^{ \pm}$on the right-hand side. Their expressions are listed in Eqs. (4.2), (4.3), (4.5), (4.6), (4.8), and (4.9), respectively.

covariant three-point vertex consisting of the basic and composite operators with the rescaled momentum operators for the integer $J$ and $s_{1,2}$ is to be presented in Sec. V.

\section{B. Fermionic vertex operators}

First, we consider the decay of a spin-1 particle $X$ into a spin-1/2 particle $M_{1}$ and a spin-1/2 antiparticle $\bar{M}_{2}$. The number of independent terms involving the $1 \rightarrow 1 / 2+1 / 2$ two-body decay is $n[1,1 / 2,1 / 2]=4$, accounting for the four reduced helicity amplitudes, $\mathcal{C}_{ \pm 1 / 2, \pm 1 / 2}^{1}$ and $\mathcal{C}_{ \pm 1 / 2, \mp 1 / 2}^{1}$. After a little manipulation, we find the following four covariant three-point operators,

$$
\begin{gathered}
P^{ \pm} \hat{k}_{\mu}=\frac{1}{2 m}\left(\eta^{-} \mp \eta^{+} \gamma_{5}\right) \hat{k}_{\mu} \leftrightarrow \mathcal{C}_{ \pm 1 / 2, \pm 1 / 2}^{1}=-\kappa, \\
W_{\mu}^{ \pm}=\frac{1}{2 \sqrt{2} m}\left(\eta^{+} \gamma_{\mu}^{+} \pm \eta^{-} \gamma_{\mu}^{-} \gamma_{5}\right) \leftrightarrow \mathcal{C}_{ \pm 1 / 2, \mp 1 / 2}^{1}=\kappa,
\end{gathered}
$$

with the abbreviated kinematic parameters $\eta^{ \pm}$and the redefined gamma matrices $\gamma_{\mu}^{ \pm}$

$$
\begin{aligned}
& \eta^{ \pm}=\sqrt{1-\left(\omega_{1} \pm \omega_{2}\right)^{2}} \text { and } \\
& \gamma_{\mu}^{ \pm}=\gamma_{\mu}+\frac{\left(\omega_{1} \pm \omega_{2}\right) \kappa}{1-\left(\omega_{1} \pm \omega_{2}\right)^{2}} \hat{k}_{\mu},
\end{aligned}
$$

satisfying the relation $\kappa=\eta^{+} \eta^{-}$. The corresponding reduced helicity amplitudes enable us to identify the basic fermionic operators, $P^{ \pm}$and $W^{ \pm}$with the normalized momentum $\hat{k}_{\mu}$ responsible for the one-half raising and lowering diagonal and antidiagonal transitions in the half-integer helicity lattice space from the point $(0,0)$ to the points $( \pm 1 / 2, \pm 1 / 2)$ and $( \pm 1 / 2, \mp 1 / 2)$, respectively, as shown in the middle panel of Fig. 2.

Second, we consider the decay $1 / 2 \rightarrow 1 / 2+1$ of a spin$1 / 2$ particle fermion $X$ into a spin- $1 / 2$ particle fermion $M_{1}$ and a spin-1 antiparticle boson $\bar{M}_{2}$. The number of independent terms involving the $1 / 2 \rightarrow 1 / 2+1$ two-body decay is $n[1 / 2,1 / 2,1]=4$, accounting for the four reduced helicity amplitudes, $\mathcal{C}_{ \pm 1 / 2,0}^{1 / 2}$ and $\mathcal{C}_{ \pm 1 / 2, \pm 1}^{1 / 2}$. After a little manipulation, we can find the following four covariant three-point operators,

$$
\begin{aligned}
P_{1}^{ \pm} \hat{p}_{2 \beta} & =\frac{1}{2 m}\left(\eta_{1}^{-} \mp \eta_{1}^{+} \gamma_{5}\right) \hat{p}_{2 \beta} \leftrightarrow \mathcal{C}_{ \pm 1 / 2,0}^{1 / 2}=-\kappa^{2}, \\
W_{1 \beta}^{ \pm} & =\frac{1}{2 \sqrt{2} m}\left(\eta_{1}^{+} \gamma_{1 \beta}^{+} \pm \eta_{1}^{-} \gamma_{1 \beta}^{-} \gamma_{5}\right) \leftrightarrow \mathcal{C}_{ \pm 1 / 2, \pm 1}^{1 / 2}=-\kappa,
\end{aligned}
$$

with the abbreviated kinematic parameters and the redefined gamma matrices $\gamma_{1 \beta}^{ \pm}$

$$
\begin{aligned}
& \eta_{1}^{ \pm}=\sqrt{\left(1 \pm \omega_{1}\right)^{2}-\omega_{2}^{2}} \text { and } \\
& \gamma_{1 \beta}^{ \pm}=\gamma_{\beta} \mp \frac{2\left(1 \pm \omega_{1}\right)}{\left(1 \pm \omega_{1}\right)^{2}-\omega_{2}^{2}} \hat{p}_{\beta},
\end{aligned}
$$

satisfying the relation $\kappa=\eta_{1}^{+} \eta_{1}^{-}$. In this case, the two fermionic operators, $P_{1}^{ \pm}$and $W_{1}^{ \pm}$with the re-scaled momentum $\hat{p}_{2 \beta}$, dictate the one-half step horizontal transitions from the point $(0,0)$ to the points $( \pm 1 / 2,0)$, and the one-half step horizontal and one-step vertical transitions from the point $(0,0)$ to the points $( \pm 1 / 2, \pm 1)$, respectively, as shown in the right panel of Fig. 2.

Properly combining all the fermionic and bosonic operators with the three rescaled momentum operators enables us to reach every half-integer helicity lattice point. To recapitulate, for any given $J$ and $s_{1,2}$, we can weave the covariant effective three-point vertex corresponding to every helicity combination of $\left[\lambda_{1}, \lambda_{2}\right]$ efficiently and systematically. The explicit form of the covariant threepoint vertex constructed by weaving the fermionic as well as bosonic operators is to be presented in Sec. V. 


\section{WEAVING THE COVARIANT THREE-POINT VERTICES}

Along with the rescaled momenta, $\hat{p}_{1,2}$ and $\hat{k}$, the four basic and two composite bosonic operators, $U_{1,2}^{ \pm}$and $U^{ \pm}$, and the two sets of basic fermionic operators, $\left\{P^{ \pm}, W^{ \pm}\right\}$ and $\left\{P_{1}^{ \pm}, W_{1}^{ \pm}\right\}$, worked out in Sec. IV, enable us to construct all the relevant covariant three-point vertices explicitly. For this construction, it is crucial to take into account the feature that bosonic and fermionic wave tensors are totally symmetric, traceless and divergence-free in their four-vector indices and the fermionic spinors satisfy

$$
\gamma_{\alpha_{i}} u_{1}^{\alpha_{1} \cdots \alpha_{i} \cdots \alpha_{n_{1}}}\left(k_{1}, \lambda_{1}\right)=\gamma_{\beta_{i}} \beta_{2}^{\beta_{1} \cdots \beta_{i} \cdots \beta_{n_{2}}}\left(k_{2}, \lambda_{2}\right)=0,
$$

as well with the non-negative integer $n_{1,2}=s_{1,2}-1 / 2$, so that every fermionic vertex involving $\gamma_{\alpha_{i}}$ or $\gamma_{\beta_{j}}$ with $i=1, \ldots, n_{1}$ and $j=1, \ldots, n_{2}$ can be effectively excluded. The $\mu, \alpha$ and $\beta$ four-vector indices in any covariant threepoint vertex can be shuffled freely due to the totally symmetric properties of the wave tensors, and any term including $p_{\mu_{i}}$ for $i=1, \cdots n$ can be excluded effectively due to the divergence-free condition. Moreover, the same condition allows us to replace $k_{2 \alpha_{i}}$ and $k_{1 \beta_{j}}$ effectively by $-p_{\alpha_{i}}$ and $p_{\beta_{j}}$ for $i=1, \ldots, n_{1}$ and $j=1, \ldots, n_{2}$.

As many indices of different types are involved in expressing a covariant three-point vertex especially for high-spin particles, we introduce the following compact square-bracket notations

$$
\begin{array}{rlrl}
{[\hat{k}]^{n} \rightarrow \hat{k}_{\mu_{1}} \cdots \hat{k}_{\mu_{n}},} & {\left[\hat{p}_{1}\right]^{n} \rightarrow \hat{p}_{1 \alpha_{1}} \cdots \hat{p}_{1 \alpha_{n}}, \quad\left[\hat{p}_{2}\right]^{n} \rightarrow \hat{p}_{2 \beta_{1}} \cdots \hat{p}_{2 \beta_{n}},} \\
{\left[U^{ \pm}\right]^{n} \rightarrow U_{\alpha_{1} \beta_{1}}^{ \pm} \cdots U_{\alpha_{n} \beta_{n}}^{ \pm},} & {\left[U_{1}^{ \pm}\right]^{n} \rightarrow U_{1 \alpha_{1} \mu_{1}}^{ \pm} \cdots U_{1 \alpha_{n} \mu_{n}}^{ \pm},} & {\left[U_{2}^{ \pm}\right]^{n} \rightarrow U_{2 \beta_{1} \mu_{1}}^{ \pm} \cdots U_{2 \beta_{n} \mu_{n}}^{ \pm},}
\end{array}
$$

for a non-negative integer $n$. Obviously, the zeroth power $(n=0)$ of any operator or rescaled four momenta is set to be 1 . We emphasize once more that any permutation of the $\alpha, \beta$ and $\mu$ four-vector indices can be regarded to be equivalent as eventually the vertex operators are to be coupled with the $X$ and $M_{1,2}$ wave tensors totally symmetric in the four-vector indices.

\section{A. Bosonic and fermionic three-point vertices}

The helicity lattice point with any specific values of $\lambda_{1}$ and $\lambda_{2}$ can be reached by the helicity-specific operators $\mathcal{H}$ consisting of the three rescaled momentum operators, $\hat{k}$ and $\hat{p}_{1,2}$ and the helicity-specific transition operators $\mathcal{T}$ constructed with a product of basic operators as

$$
\begin{aligned}
{\left[\mathcal{H}_{A\left[\lambda_{1}, \lambda_{2}\right]}^{J, s_{1}, s_{2}}\right]=} & {[\hat{k}]^{J-\left|\lambda_{1}-\lambda_{2}\right|}\left[\hat{p}_{1}\right]^{s_{1}-\left|\lambda_{1}\right|}\left[\hat{p}_{2}\right]^{s_{2}-\left|\lambda_{2}\right|}\left[\mathcal{T}_{A\left[\lambda_{1}, \lambda_{2}\right]}^{J, s_{1}, s_{2}}\right] } \\
& \text { with } \quad\left|\lambda_{1}-\lambda_{2}\right| \leq J
\end{aligned}
$$

in an operator form where the index $A=i i i$, ihh, and $h h i$ indicates whether the spins of $X$ and $M_{1,2}$ are integer $(i)$ or half-integer $(h)$, respectively. For mathematical consistency, the powers of the rescaled momentum operators, $\hat{p}_{1,2}$ and $\hat{k}$, should be non-negative and they play a crucial role in determining the number of independent terms.

The helicity-specific operators in Eq. (5.4) can be applied even to the massless case with $m_{1}=0$ or $m_{2}=0$ simply by setting $\left[\hat{p}_{1}\right]^{s_{1}-\left|\lambda_{1}\right|}$ or $\left[\hat{p}_{2}\right]^{s_{2}-\left|\lambda_{2}\right|}$ to unity, because only the maximal helicity values identical to the spin in magnitude are allowed physically for a massless particle.

First, in the iii case with an integer $s_{1}$ and an integer $s_{2}$ forcing the spin $J$ to be an integer, the transition vertices consisting of a sequence of the bosonic scalar and vector operators, $U^{ \pm}$and $U_{1,2}^{ \pm}$, are classified by three regions as

$$
\left[\mathcal{T}_{i i i\left[\lambda_{1}, \lambda_{2}\right]}^{J, s_{1}, s_{2}}\right]= \begin{cases}{\left[U^{ \pm}\right]^{\left|\lambda_{2}\right|}\left[U_{1}^{ \pm}\right]^{\left|\lambda_{1}-\lambda_{2}\right|}} & \text { for } \lambda_{1,2}= \pm\left|\lambda_{1,2}\right| \text { and } 0<\left|\lambda_{2}\right| \leq\left|\lambda_{1}\right|, \\ {\left[U^{ \pm}\right]^{\left|\lambda_{1}\right|}\left[U_{2}^{ \pm}\right]^{\left|\lambda_{1}-\lambda_{2}\right|}} & \text { for } \lambda_{1,2}= \pm\left|\lambda_{1,2}\right| \text { and } 0<\left|\lambda_{1}\right|<\left|\lambda_{2}\right|, \\ {\left[U_{1}^{ \pm}\right]^{\left|\lambda_{1}\right|}\left[U_{2}^{\mp}\right]^{\left|\lambda_{2}\right|}} & \text { for } \lambda_{1}= \pm\left|\lambda_{1}\right| \text { and } \lambda_{2}=\mp\left|\lambda_{2}\right|\end{cases}
$$

in an operator form.

Second, in the ihh case with a half-integer $s_{1}$ and a half-integer $s_{2}$ forcing the spin $J$ to be an integer, the transition vertices consisting of the fermionic operators $P^{ \pm}$and $W^{ \pm}$as well as the bosonic scalar and vector operators, $U^{ \pm}$and $U_{1,2}^{ \pm}$, are classified as

$$
\left[\mathcal{T}_{i h h\left[\lambda_{1}, \lambda_{2}\right]}^{J, s_{1}, s_{2}}\right]= \begin{cases}{\left[P^{ \pm}\right]\left[U^{ \pm}\right]^{\left|\lambda_{2}\right|-1 / 2}\left[U_{1}^{ \pm}\right]^{\left|\lambda_{1}-\lambda_{2}\right|}} & \text { for } \lambda_{1,2}= \pm\left|\lambda_{1,2}\right| \text { and }\left|\lambda_{2}\right| \leq\left|\lambda_{1}\right|, \\ {\left[P^{ \pm}\right]\left[U^{ \pm}\right]^{\left|\lambda_{1}\right|-1 / 2}\left[U_{2}^{ \pm}\right]^{\left|\lambda_{1}-\lambda_{2}\right|}} & \text { for } \lambda_{1,2}= \pm\left|\lambda_{1,2}\right| \text { and }\left|\lambda_{1}\right|<\left|\lambda_{2}\right|, \\ {\left[W^{ \pm}\right]\left[U_{1}^{ \pm}\right]^{\left|\lambda_{1}\right|-1 / 2}\left[U_{2}^{\mp}\right]^{\left|\lambda_{2}\right|-1 / 2}} & \text { for } \lambda_{1}= \pm\left|\lambda_{1}\right| \text { and } \lambda_{2}=\mp\left|\lambda_{2}\right|,\end{cases}
$$

in an operator form. 
Third, in the hhi case with a half-integer $s_{1}$ and an integer $s_{2}$ forcing the spin $J$ to be a half-integer, the transition vertices consisting of the fermionic operators $P_{1}^{ \pm}$and $W_{1}^{ \pm}$as well as the bosonic scalar and vector operators, $U^{ \pm}$and $U_{1,2}^{ \pm}$, are given by

$$
\left[\mathcal{T}_{\text {hhi }\left[\lambda_{1}, \lambda_{2}\right]}^{J, s_{1}, s_{2}}\right]= \begin{cases}{\left[P_{1}^{ \pm}\right]\left[U^{ \pm}\right]^{\left|\lambda_{2}\right|}\left[U_{1}^{ \pm}\right]^{\left|\lambda_{1}-\lambda_{2}\right|-1 / 2}} & \text { for } \lambda_{1,2}= \pm\left|\lambda_{1,2}\right| \text { and } 1<\left|\lambda_{2}\right|<\left|\lambda_{1}\right|, \\ {\left[W_{1}^{ \pm}\right]\left[U^{ \pm}\right]^{\left|\lambda_{1}\right|-1 / 2}\left[U_{2}^{ \pm}\right]^{\left|\lambda_{1}-\lambda_{2}\right|-1 / 2}} & \text { for } \lambda_{1,2}= \pm\left|\lambda_{1,2}\right| \text { and }\left|\lambda_{1}\right|<\left|\lambda_{2}\right|, \\ {\left[P_{1}^{ \pm}\right]\left[U_{1}^{ \pm}\right]^{\left|\lambda_{1}\right|-1 / 2}\left[U_{2}^{\mp}\right]^{\left|\lambda_{2}\right|}} & \text { for } \lambda_{1}= \pm\left|\lambda_{1}\right| \text { and } \lambda_{2}=\mp\left|\lambda_{2}\right|,\end{cases}
$$

in an operator form.

To conclude, the general form of any covariant three-point vertex $\Gamma_{A \alpha \beta ; \mu}$ for any given $J$ and $s_{1,2}$ with $A=i i i, i h h$, and hhi is a linear combination of all the allowed helicityspecific three-point vertices. The succinct operator form of the covariant three-point vertex is given by

$$
\begin{aligned}
{\left[\Gamma_{A}\right]=} & \sum_{\lambda_{1}=-s_{1}}^{s_{1}} \sum_{\lambda_{2}=-s_{2}}^{s_{2}} c_{A\left[\lambda_{1}, \lambda_{2}\right]}^{J, s_{1}, s_{2}}\left[\mathcal{H}_{A\left[\lambda_{1}, \lambda_{2}\right]}^{J, s_{1}, s_{2}}\right] \\
& \text { with } \quad A=\text { iii, ihh, hhi, }
\end{aligned}
$$

with the constraint $\left|\lambda_{1}-\lambda_{2}\right| \leq J$ where the helicity-specific coefficients, $c_{A\left[\lambda_{1}, \lambda_{2}\right]}^{J, s_{1}, s_{2}}$ with $A=i i i, i h h$, or $h h i$ depend only on the three masses, $m$ and $m_{1,2}$. The expression (5.8) along with the helicity-specific covariant transition vertex operators in Eqs. (5.5), (5.6), and (5.7) is the key result of the present work. Although it is originally deduced from the comparison with the helicity amplitudes in the XRF, the form is valid in every reference frame because of its Lorentz-covariant form.

\section{B. Conversion to all the other helicity-specific vertices}

So far, we have derived the explicit forms of the helicity-specific operators only for the spin combinations, (iii), $(i h h)$, and $(h h i)$ in Sec. VA. However, the following simple symmetry arguments enable us to obtain the covariant three-point vertices for all the remaining spin assignments.

Since any integer-spin wave tensor is given in the same form regardless of whether the state is treated as a particle or an antiparticle, it is unnecessary to consider the conversion of the helicity-specific operators $\left[\mathcal{H}_{i i i\left[\lambda_{1}, \lambda_{2}\right]}^{J, s_{1}, s_{2}}\right]$. In contrast, the helicity-specific operators $\left[\overline{\mathcal{H}}_{i h h\left[\lambda_{1}, \lambda_{2}\right]}^{J, s_{1}, s_{2}}\right]$ for the decay of a integer spin- $J$ particle $X$ into an antiparticle $\bar{M}_{1}$ and a particle $M_{2}$ of half-integer spins $s_{1,2}$ can be given in an operator form by

$$
\left[\overline{\mathcal{H}}_{i h h\left[\lambda_{1}, \lambda_{2}\right]}^{J, s_{1}, s_{2}}\right]=-\left[C \mathcal{H}_{i h h\left[\lambda_{1}, \lambda_{2}\right]}^{J, s_{1}, s_{2}} C^{-1}\right] .
$$

This relation is derived by converting the wave spinors $\bar{u}_{1}^{\alpha}$ and $v_{2}^{\beta}$ of $M_{1}$ and $\bar{M}_{2}$ to $v_{1}^{\alpha}$ and $\bar{u}_{2}^{\beta}$ of $\bar{M}_{1}$ and $M_{2}$ in the existing helicity-specific amplitudes by the unitary chargeconjugation $C$ giving the relation $v=C \bar{u}^{T}$. Similarly, the helicity-specific operators $\left[\overline{\mathcal{H}}_{h h i\left[\lambda_{1}, \lambda_{2}\right]}^{J, s_{1}, s_{2}}\right]$ for the decay of a half-integer spin- $J$ antiparticle $\bar{X}$ into a half-integer spin- $s_{1}$ antiparticle $\bar{M}_{1}$ and a integer spin- $s_{2}$ particle $M_{2}$ is obtained by the relation

$$
\left[\overline{\mathcal{H}}_{\left.h h i \lambda_{1}, \lambda_{2}\right]}^{J, s_{1}, s_{2}}\right]=-\left[C \mathcal{H}_{h h i\left[\lambda_{1}, \lambda_{2}\right]}^{J, s_{1}, s_{2}} C^{-1}\right]
$$

in an operator form by converting the wave spinors $\bar{u}_{1}^{\alpha}$ and $u_{\mu}$ to $v_{1}^{\alpha}$ and $\bar{v}_{\mu}$ with the charge-conjugation $C$.

On the other hand, the helicity-specific operators for the decay of a half-integer spin- $J$ particle $X$ of mass $m$ and helicity $\sigma$ into an integer spin- $s_{2}$ antiparticle $\bar{M}_{1}$ and a halfinteger spin- $s_{1}$ particle $M_{2}$ of masses $m_{1,2}$ and helicities $\lambda_{2,1}$, respectively, are obtained simply by the relation

$$
\begin{aligned}
& \left(\mathcal{H}_{h i h\left[\lambda_{2}, \lambda_{1}\right]}^{J, s_{2}, s_{1}}\right)_{\alpha_{1} \cdots \alpha_{n_{2}}, \beta_{1} \cdots \beta_{n_{1}}}^{\mu_{1} \cdots \mu_{n}}(p, q) \\
& =\left(\mathcal{H}_{\text {hhi }\left[\lambda_{1}, \lambda_{2}\right]}^{J, s_{1}, s_{2}}\right)_{\beta_{1} \cdots \beta_{n_{1}}, \alpha_{1} \cdots \alpha_{n_{2}}}^{\mu_{1}}(p,-q),
\end{aligned}
$$

through the replacements of $k_{1} \leftrightarrow k_{2}, \quad \alpha_{1} \cdots \alpha_{n_{1}} \rightarrow$ $\beta_{1} \cdots \beta_{n_{1}}$, and $\beta_{1} \cdots \beta_{n_{2}} \rightarrow \alpha_{1} \cdots \alpha_{n_{2}}$.

\section{Off-shell electromagnetic gauge-invariant vertices}

Due to the electromagnetic (EM) gauge invariance, any off-shell photon couples to a conserved current. Therefore, in any timelike photon exchange process involving the $\gamma^{*} M_{1} \bar{M}_{2}$ vertex, the off-shell photon can be treated as a spin-1 particle of mass $m=\sqrt{p^{2}}$. Moreover, the covariant three-point $\gamma^{*} M_{1} \bar{M}_{2}$ vertex can be cast into a manifestly EM gauge-invariant form [14] as

$$
\Gamma_{X \mathrm{EM} \alpha, \beta}^{\mu}=p^{2} \Gamma_{\alpha, \beta}^{\mu}-\left(p \cdot \Gamma_{\alpha, \beta}\right) p^{\mu},
$$

satisfying the current conservation condition $p_{\mu} \Gamma_{X \mathrm{EM} \alpha, \beta}^{\mu}=$ $p \cdot \Gamma_{X \mathrm{EM} \alpha, \beta}=0$ automatically.

Similarly, in any process involving the $X \gamma^{*} \bar{M}_{2}$ vertex, the off-shell photon $\gamma^{*}$ can be treated as a spin-1 particle of mass $m_{1}=\sqrt{k_{1}^{2}}$. Then, the covariant three-point vertex can be cast into a manifestly EM gauge-invariant form as

$$
\Gamma_{1 \mathrm{EM} \alpha, \beta}^{\mu}=k_{1}^{2} \Gamma_{\alpha, \beta}^{\mu}-k_{1}^{\rho} k_{1 \alpha} \Gamma_{\rho, \beta}^{\mu},
$$


satisfying the current conservation condition $k_{1}^{\alpha} \Gamma_{1 \mathrm{EM} \alpha, \beta}^{\mu}=0$ automatically.

\section{CONCLUSIONS}

We have developed an efficient algorithm for constructing all the covariant effective three-point vertices for the decay of a particle $X$ of spin $J$ and mass $m$ into a particle $M_{1}$ and an antiparticle $\bar{M}_{2}$ with any spins and masses, $s_{1,2}$ and $m_{1,2}$. For this development, we have exploited the closely related equivalence between the helicity formalism and the covariant formulation for identifying the basic operators and then for constructing all the covariant threepoint vertices.

We have presented all the helicity-specific covariant threepoint vertices in an operator form in Eqs. (5.5), (5.6), and (5.7) explicitly in the $i i i, i h h$, and $h h i$ cases, respectively. We have listed the conversion rules to all the other cases in Eqs. (5.9), (5.10), and (5.11). In addition, we have shown that the case with $m_{1}=0$ or $m_{2}=0$ can be accommodated straightforwardly. Finally, we have described how to obtain the EM gauge-invariant vertices involving a virtual photon in the initial or final state.

The general algorithm for constructing the covariant three-point vertices enables us to work out various theoretical and phenomenological aspects systematically and efficiently, including the indirect and direct searches for DM particles of any spin and the production of new particles of any spin at high energy colliders. An interesting issue to be pursued is whether the bosonic and fermionic cases can be synthesized in a more compact way.

\section{ACKNOWLEDGMENTS}

The work was in part by the Basic Science Research Program of Ministry of Education through National Research Foundation of Korea (Grant No. NRF2016R1D1A3B01010529) and in part by the CERNKorea theory collaboration.
[1] S. L. Glashow, Nucl. Phys. 22, 579 (1961); S. Weinberg, Phys. Rev. Lett. 19, 1264 (1967); A. Salam, Conf. Proc. C 680519, 367 (1968); H. Fritzsch, M. Gell-Mann, and H. Leutwyler, Phys. Lett. 47B, 365 (1973).

[2] G. Aad et al. (ATLAS Collaboration), Phys. Lett. B 716, 1 (2012); S. Chatrchyan et al. (CMS Collaboration), Phys. Lett. B 716, 30 (2012).

[3] P. A. Zyla et al. (Particle Data Group), Prog. Theor. Exp. Phys. (2020), 083C01.

[4] P. Nath and R. L. Arnowitt, Phys. Lett. 56B, 177 (1975); D. V. Volkov and V. A. Soroka, JETP Lett. 18, 312 (1973);

D. Z. Freedman, P. van Nieuwenhuizen, and S. Ferrara, Phys. Rev. D 13, 3214 (1976); S. Deser and B. Zumino, Phys. Lett. 62B, 335 (1976).

[5] B. P. Abbott et al. (LIGO Scientific and Virgo Collaborations), Phys. Rev. Lett. 116, 061102 (2016).

[6] I. Antoniadis, N. Arkani-Hamed, S. Dimopoulos, and G. R. Dvali, Phys. Lett. B 436, 257 (1998); N. Arkani-Hamed, S. Dimopoulos, and G. R. Dvali, Phys. Lett. B 429, 263 (1998); L. Randall and R. Sundrum, Phys. Rev. Lett. 83, 3370 (1999).

[7] E. Babichev, L. Marzola, M. Raidal, A. Schmidt-May, F. Urban, H. Veermäe, and M. von Strauss, Phys. Rev. D 94, 084055 (2016); E. Babichev, L. Marzola, M. Raidal, A. Schmidt-May, F. Urban, H. Veermäe, and M. von Strauss, J. Cosmol. Astropart. Phys. 09 (2016) 016; L. Marzola,
M. Raidal, and F. R. Urban, Phys. Rev. D 97, 024010 (2018); J. C. Criado, N. Koivunen, M. Raidal, and H. Veermäe, Phys. Rev. D 102, 125031 (2020); A. Falkowski, G. Isabella, and C. S. Machado, SciPost Phys. 10, 101 (2021); P. Gondolo, S. Kang, S. Scopel, and G. Tomar, Phys. Rev. D 104, 063017 (2021).

[8] S. Y. Choi and J.H. Jeong, Phys. Rev. D 103, 096013 (2021).

[9] S. Y. Choi and J.H. Jeong, Phys. Rev. D 104, 055046 (2021).

[10] R. E. Behrends and C. Fronsdal, Phys. Rev. 106, 345 (1957); P. R. Auvil and J. J. Brehm, Phys. Rev. 145, 1152 (1966); P. J. Caudrey, I. J. Ketley, and R. C. King, Nucl. Phys. B6, 671 (1968); M. D. Scadron, Phys. Rev. 165, 1640 (1968); S. U. Chung, Phys. Rev. D 57, 431 (1998); S. Z. Huang, T. N. Ruan, N. Wu, and Z. P. Zheng, Eur. Phys. J. C 26, 609 (2003).

[11] M. Jacob and G. C. Wick, Ann. Phys. (N.Y.) 7, 404 (1959).

[12] G. Durieux, T. Kitahara, C. S. Machado et al., J. High Energy Phys. 12 (2020) 175; N. Arkani-Hamed, T. C. Huang, and Y. T. Huang, J. High Energy Phys. 11 (2021) 70.

[13] M. E. Rose, Elementary Theory of Angular Momentum (Dover Publication Inc., New York, 2011), ISBN-13:9780486684802.

[14] F. Boudjema and C. Hamzaoui, Phys. Rev. D 43, 3748 (1991). 